\title{
Kringle Domain
}

National Cancer Institute

\section{Source}

National Cancer Institute. Kringle Domain. NCI Thesaurus. Code C13290.

Kringles are autonomous structural domains, found throughout the blood clotting and fibrinolytic proteins. Kringle domains are believed to play a role in binding mediators (e.g., membranes, other proteins or phospholipids), and in the regulation of proteolytic activity. Kringle domains are characterised by a triple loop, 3-disulphide bridge structure, whose conformation is defined by a number of hydrogen bonds and small pieces of anti-parallel beta-sheet. They are found in a varying number of copies, in some serine proteases and plasma proteins. 